Yukawa Institute Kyoto

YITP-03-50

quant-ph/0308040

August 2003

\title{
Quantum vs Classical Mechanics: role of elementary excitations
}

\author{
I. Loris ${ }^{a, b}$ and R. Sasaki ${ }^{a}$ \\ ${ }^{a}$ Yukawa Institute for Theoretical Physics, \\ Kyoto University, Kyoto 606-8502, Japan \\ ${ }^{b}$ Dienst Theoretische Natuurkunde, Vrije Universiteit Brussel, \\ Pleinlaan 2, B-1050 Brussels, Belgium
}

\begin{abstract}
Simple theorems relating a quantum mechanical system to the corresponding classical one at equilibrium and connecting the quantum eigenvalues to the frequencies of normal modes oscillations are presented. Corresponding to each quantum eigenfunction, a 'classical eigenfunction' is associated. Those belonging to 'elementary excitations' play an important role.
\end{abstract}

\section{Introduction}

In this paper we will address a general and a most fundamental issue in multi-particle quantum mechanics; the correspondence/contrast between quantum and classical mechanics. Usually such correspondence/contrast is discussed in the "quasi-classical" or "quasimacroscopic" regime of quantum mechanics in which the expectation values are good representations of the classical variables. These are exemplified in Ehrenfest's well-known theorem or in the WKB method. Here we ask a rather different question. Suppose a multi-particle quantum mechanical system has a unique ground state and discrete energy spectrum. We naturally expect that the corresponding classical potential has a well-defined minimum, 
which gives an equilibrium point. Near the equilibrium, the system is reduced to a collection of harmonic oscillators as many as the degrees of freedom.

We will ask and answer in simple terms the following universal question in multi-particle quantum mechanics:

How can we relate the knowledge of the eigenfunctions and eigenvalues of a multi-particle quantum mechanical system to the properties of the corresponding classical system, in particular, at equilibrium?

In fact we show that for each quantum eigenfunction a corresponding "classical eigenfunction" is defined as the $\hbar \rightarrow 0$ limit, see (16). These classical eigenfunctions satisfy simple eigenvalue equations (17). Among them, there are "elementary excitations", as many as the degrees of freedom, corresponding to each normal mode of the small oscillations at equilibrium (20). They are the generators of the classical eigenfunctions. The quantum eigenfunctions are the $\hbar$-deformations of these classical eigenfunctions. We show that the main part of the generic quantum eigenvalues, which is proportional to Planck's constant $\hbar$, is given by a linear combination of the (angular) frequencies of small oscillations with integer coefficients (22).

Another motivation of the present research is to provide an analytical proof for the recent results on the quantum vs classical integrability in Calogero-Moser (C-M) systems by Corrigan-Sasaki [1]. C-M systems [2] are classical and quantum integrable multi-particle dynamics based on root systems and the quantum eigenvalues are expressed in terms of roots and weights. In other words, they have 'integer' energy spectra. It was shown by direct numerical calculation [1] that most of the classical data, for example, the (angular) frequencies of small oscillations at equilibrium are also 'integers'. The Propositions 1-3 in section 2 give a simple analytic proof for these interesting observations. Thanks to the integrability (exact solvability), classical and quantum eigenfunctions for C-M systems based on any root system can be constructed explicitly. They will be shown in a subsequent paper [3] in some details, in particular, those for the elementary excitations. These provide excellent explicit examples of the main results of this paper.

The Planck's constant $\hbar$ is always written explicitly in this article. This paper is organised as follows. In section 2, the formulation of multi-particle quantum mechanics in terms of the prepotential is introduced and the basic results on the quantum and classical eigenfunctions are derived in an elementary way. Section 3 is devoted for summary and comments. 


\section{Multi-particle Quantum Mechanics}

We will discuss a multi-particle quantum mechanical system and its relationship with the corresponding classical $(\hbar \rightarrow 0)$ dynamics. The dynamical variables are the coordinates $\left\{q_{j} \mid j=1, \ldots, r\right\}$ and their canonically conjugate momenta $\left\{p_{j} \mid j=1, \ldots, r\right\}$, subject to the Heisenberg commutation relations or the Poisson bracket relations. We will adopt the standard vector notation in $\mathbb{R}^{r}$ :

$$
q=\left(q_{1}, \ldots, q_{r}\right), \quad p=\left(p_{1}, \ldots, p_{r}\right), \quad q^{2} \equiv \sum_{j=1}^{r} q_{j}^{2}, \quad p^{2} \equiv \sum_{j=1}^{r} p_{j}^{2}, \ldots,
$$

in which $r$ is the number of particles. In quantum theory, the momentum operator $p_{j}$ acts as a differential operator:

$$
p_{j}=-i \hbar \frac{\partial}{\partial q_{j}}, \quad j=1, \ldots, r
$$

Throughout this paper we discuss the standard Hamiltonian system

$$
H=\frac{1}{2} p^{2}+V(q)
$$

in which we have assumed for simplicity that all the particles have the same mass, which is rescaled to unity. Let us start with mild assumptions that the system has a unique and square integrable ground state $\psi_{0}$ :

$$
H \psi_{0}=0, \quad \int\left|\psi_{0}\right|^{2} d^{r} q<\infty, \quad E_{0}=0,
$$

and that it has a finite (or an infinite) number of discrete eigenvalues:

$$
H \psi_{n}=E_{n} \psi_{n}, \quad E_{n}=\mathcal{E}_{n} \hbar+\mathcal{O}\left(\hbar^{2}\right)
$$

Here we adopt the convention that the ground state energy is vanishing $E_{0}=0$, by adjusting the constant part of the potential $V$, see below.

Since the above time-independent Schrödinger equation is real for a self-adjoint Hamiltonian and that the ground state has no node we express the ground state eigenfunction as

$$
\psi_{0}(q)=e^{\frac{1}{\hbar} W(q)}
$$

in which a real function $W=W(q)$ is called a prepotential 4]. By simple differentiation of (41), we obtain

$$
p_{j} \psi_{0}=-i \frac{\partial W}{\partial q_{j}} \psi_{0}, \quad p^{2} \psi_{0}=-\sum_{j=1}^{r}\left[\left(\frac{\partial W}{\partial q_{j}}\right)^{2}+\hbar \frac{\partial^{2} W}{\partial q_{j}^{2}}\right] \psi_{0}
$$


which results in

$$
\left\{\frac{1}{2} p^{2}+\frac{1}{2} \sum_{j=1}^{r}\left[\left(\frac{\partial W}{\partial q_{j}}\right)^{2}+\hbar \frac{\partial^{2} W}{\partial q_{j}^{2}}\right]\right\} \psi_{0}=0 .
$$

In other words, we can express the Hamiltonian and the potential in terms of the prepotential [1, 4] ${ }^{1}$

$$
H(W)=\frac{1}{2} p^{2}+V(q), \quad V(q)=\frac{1}{2} \sum_{j=1}^{r}\left[\left(\frac{\partial W}{\partial q_{j}}\right)^{2}+\hbar \frac{\partial^{2} W}{\partial q_{j}^{2}}\right] .
$$

By removing the obvious $\hbar$-dependent terms, let us define a classical potential $V_{C}(q)$ :

$$
V_{C}(q)=\frac{1}{2} \sum_{j=1}^{r}\left(\frac{\partial W}{\partial q_{j}}\right)^{2}
$$

Equivalently one could introduce the classical Hamiltonian $H_{C}$ as an 'average' of the original Hamiltonian $H(W)$ with the one whose ground state is the inverse of the original ground state $^{2} H(-W)$ :

$$
H_{C}=(H(W)+H(-W)) / 2=\frac{1}{2} p^{2}+V_{C}(q) .
$$

Conversely, (77) is a Riccati equation determining the prepotential $W$ for a given potential $V$ (or $V_{C}$ ). Needless to say, it does not matter if the prepotential can be expressed in terms of elementary functions or not.

\subsection{Equilibrium position and frequencies of small oscillations}

Now let us consider the equilibrium point of the classical potential $V_{C}$ (8). The classical Hamiltonian (9) has a stationary solution at the classical equilibrium point, $p=0, q=\bar{q}$. There could be, in general, many stationary points of the classical potential $V_{C}$, among which we will focus on the 'maximum' point $\bar{q}$ of the ground state wavefunction $\psi_{0}$ [1]:

$$
\frac{\partial W(\bar{q})}{\partial q_{j}}=0, \quad \Longrightarrow \frac{\partial V_{C}(\bar{q})}{\partial q_{j}}=\sum_{k=1}^{r} \frac{\partial^{2} W(\bar{q})}{\partial q_{j} \partial q_{k}} \frac{\partial W(\bar{q})}{\partial q_{k}}=0, \quad j=1, \ldots, r
$$

By expanding the classical potential $V_{C}$ around $\bar{q}$, we obtain

$$
\begin{aligned}
V_{C}(q) & =\frac{1}{2} \sum_{j, k=1}^{r} \frac{\partial^{2} V_{C}(\bar{q})}{\partial q_{j} \partial q_{k}}(q-\bar{q})_{j}(q-\bar{q})_{k}+\mathcal{O}\left((q-\bar{q})^{3}\right) \\
& =\frac{1}{2} \sum_{j, k, l=1}^{r} \frac{\partial^{2} W(\bar{q})}{\partial q_{j} \partial q_{l}} \frac{\partial^{2} W(\bar{q})}{\partial q_{l} \partial q_{k}}(q-\bar{q})_{j}(q-\bar{q})_{k}+\mathcal{O}\left((q-\bar{q})^{3}\right)
\end{aligned}
$$

\footnotetext{
${ }^{1}$ Similar formulas can be found within the context of supersymmetric quantum mechanics [6]. Here we stress that supersymmetry is not necessary.

${ }^{2}$ This is the main ingredient of the well-known Darboux transformation [7].
} 
since $V_{C}(\bar{q})=0$, (마). Thus the eigen (angular) frequencies (frequency squared) of small oscillations near the classical equilibrium are given as the eigenvalues of the Hessian matrix $\widetilde{W}\left(\widetilde{V}_{C}\right)$ :

$$
\widetilde{W}=\operatorname{Matrix}\left[\frac{\partial^{2} W(\bar{q})}{\partial q_{j} \partial q_{k}}\right], \quad \widetilde{V}_{C}=\text { Matrix }\left[\frac{\partial^{2} V_{C}(\bar{q})}{\partial q_{j} \partial q_{k}}\right]=\widetilde{W}^{2}
$$

\subsection{Quantum \& Classical Eigenfunctions}

Let us express the discrete eigenfunctions in product forms

$$
\psi_{n}(q)=\phi_{n}(q) \psi_{0}(q), \quad n=0,1, \ldots, \quad \phi_{0} \equiv 1
$$

in which $\phi_{n}$ obeys a simplified equation with the similarity transformed Hamiltonian $\tilde{H}$ [4]

$$
\begin{aligned}
\tilde{H} \phi_{n} & =E_{n} \phi_{n} \\
\tilde{H}=e^{-\frac{1}{\hbar} W} H e^{\frac{1}{\hbar} W} & =-\frac{\hbar^{2}}{2} \sum_{j=1}^{r} \frac{\partial^{2}}{\partial q_{j}^{2}}-\hbar \sum_{j=1}^{r} \frac{\partial W}{\partial q_{j}} \frac{\partial}{\partial q_{j}}
\end{aligned}
$$

Here we adjust the normalisation of the eigenfunctions $\left\{\phi_{n}\right\}$ so that the corresponding "classical" eigenfunctions $\left\{\varphi_{n}\right\}$ are finite (non-vanishing) in the limit $\hbar \rightarrow 0$ :

$$
\lim _{\hbar \rightarrow 0} \phi_{n}(q)=\varphi_{n}(q), \quad n=1,2, \ldots,
$$

By taking the classical limit $(\hbar \rightarrow 0)$ of (14) and considering (13), (15), we arrive at an 'eigenvalue equation' for the "classical" wavefunctions

$$
-\sum_{j=1}^{r} \frac{\partial W}{\partial q_{j}} \frac{\partial \varphi_{n}}{\partial q_{j}}=\mathcal{E}_{n} \varphi_{n}, \quad n=1,2, \ldots,
$$

Conversely one could define the classical eigenfunctions as solutions of the above eigenvalue equation. In this case the classical eigenfunctions must satisfy certain regularity conditions. Then the quantum eigenfunction $\phi_{n}$ could be considered as an $\hbar$-deformation of the classical eigenfunction $\varphi_{n}$. For the Calogero and Sutherland systems to be discussed in a subsequent paper [3], there is a one-to-one correspondence between the classical and quantum eigenfunctions. For generic multi-particle quantum mechanical systems, the situation is less clear. 


\subsection{Main Results}

The classical eigenfunctions have the following remarkable properties:

Proposition 1 The product of two classical eigenfunctions $\left(\varphi_{n}, \mathcal{E}_{n}\right)$ and $\left(\varphi_{m}, \mathcal{E}_{m}\right)$ is again a classical eigenfunction with the eigenvalue $\mathcal{E}_{n}+\mathcal{E}_{m}$,

$$
-\sum_{j=1}^{r} \frac{\partial W}{\partial q_{j}} \frac{\partial\left(\varphi_{n} \varphi_{m}\right)}{\partial q_{j}}=\left(\mathcal{E}_{n}+\mathcal{E}_{m}\right) \varphi_{n} \varphi_{m}
$$

Proposition 2 The classical eigenfunctions vanish at the equilibrium $\bar{q}$

$$
\varphi_{n}(\bar{q})=0, \quad n=1,2, \ldots,
$$

Proposition 3 The derivatives of a classical eigenfunction at the equilibrium $\bar{q}$ form an eigenvector of the Hessian matrix $\widetilde{W}$, iff $\left.\nabla \varphi_{n}\right|_{\bar{q}} \neq 0$

$$
\begin{aligned}
-\left.\widetilde{W} \cdot \nabla \varphi_{n}\right|_{\bar{q}} & =\left.\mathcal{E}_{n} \nabla \varphi_{n}\right|_{\bar{q}}, \quad n=1,2, \ldots, . \\
& \text { or } \\
-\sum_{j=1}^{r} \frac{\partial^{2} W(\bar{q})}{\partial q_{k} \partial q_{j}} \frac{\partial \varphi_{n}(\bar{q})}{\partial q_{j}} & =\mathcal{E}_{n} \frac{\partial \varphi_{n}(\bar{q})}{\partial q_{k}}, \quad n=1,2, \ldots, .
\end{aligned}
$$

Obviously the Hessian matrix $\widetilde{W}(12)$ has at most $r$ different eigenvalues and eigenvectors. The classical eigenfunctions $\left\{\left(\varphi_{j}, \mathcal{E}_{j}\right)\right\}, j=1, \ldots, r$ for which $\left.\nabla \varphi_{j}\right|_{\bar{q}} \neq 0$ will be called "elementary excitations". At equilibrium, each corresponds to the normal coordinate of the small oscillations with the eigen (angular) frequency $\mathcal{E}_{j}$. That is, the 'main part' $\mathcal{E}_{n}$ (i.e, $\mathcal{O}(\hbar)$ part) (3i) of the quantum energy eigenvalue $E_{n}$ is given by the classic eigenfrequencies of the normal mode oscillations at the classical equilibrium. The elementary excitations are the generators of the classical eigenfunctions. In other words, any classical eigenfunction can be expressed as

$$
\varphi_{1}^{n_{1}} \cdots \varphi_{r}^{n_{r}}, \quad \mathcal{E}=n_{1} \mathcal{E}_{1}+\cdots+n_{r} \mathcal{E}_{r}, \quad n_{j} \in \mathbb{Z}_{+},
$$

or a linear combination thereof with the same eigenvalue $\mathcal{E}$. The above type of classical eigenfunctions are obviously non-elementary and they have zero gradient at equilibrium, for example, $\left.\nabla\left(\varphi_{j} \varphi_{k}\right)\right|_{\bar{q}}=0$. 


\section{Summary and comments}

We have shown that for any multi-particle quantum mechanical system,

the main part i.e. the $\mathcal{O}(\hbar)$ part, of the quantum energy eigenvalue is determined solely by the corresponding classical data, i.e. the eigenfrequencies of the normal mode oscillations at the classical equilibrium.

This is a very powerful result, since for most multi-particle systems the quantum eigenvalues are hard to evaluate, whereas the eigenfrequencies of the normal mode oscillations at classical equilibrium are easily calculated. The Calogero-Moser (C-M) systems based on any root system [2] provide ideal explicit examples in which the above Propositions 1-3 are thoroughly verified in Corrigan-Sasaki paper [1. Thanks to the exact solvability, all the quantum eigenvalues of the C-M systems are known [4, 5] and they are compared with the eigenfrequencies of the normal mode oscillations at classical equilibrium evaluated in [1]. The classical and quantum eigenfunctions for the elementary excitations are reported in some detail in Loris-Sasaki paper [3]. It should be mentioned that Perelomov's recent work 9] asserts essentially our Proposition 3 for the special cases of the quantum-classical eigenvalue correspondence of the Sutherland systems.

Let us present a few elementary examples of one degree of freedom quantum mechanics to illustrate the prepotential method and the main results explicitly.

Harmonic oscillator The ground state wavefunction, the prepotential, the quantum and classical potential, etc are $(\omega>0)$ :

$$
\psi_{0}=e^{-\omega q^{2} / 2 \hbar}, \quad W=-\omega q^{2} / 2, \quad V(q)=\omega^{2} q^{2} / 2-\omega \hbar / 2, \quad V_{C}(q)=\omega^{2} q^{2} / 2
$$

The quantum eigenvalues and eigenfunctions are:

$$
E_{n}=n \hbar \omega, \quad \mathcal{E}_{n}=n \omega, \quad \phi_{n}(q)=H_{n}(\sqrt{\omega / \hbar} q),
$$

in which $H_{n}$ is the Hermite polynomial. The classical equilibrium point is the origin $\bar{q}=0$

and the classical eigenfunction and the Hessian $\widetilde{W}(12)$ are:

$$
\varphi_{n}(q)=\lim _{\hbar \rightarrow 0} \hbar^{n / 2} H_{n}(\sqrt{\omega / \hbar} q)=\omega^{n / 2} q^{n}, \quad-\widetilde{W}=\omega .
$$

It is trivial to check (17) and the Propositions 1-3.

'Soliton' potential Let us consider a simple Pöschl-Teller [8] potential

$$
\psi_{0}=1 /(\cosh q)^{g / \hbar}, \quad W=-g \log \cosh q
$$




$$
V(q)=-\frac{g(g+\hbar)}{2 \cosh ^{2} q}+\frac{g^{2}}{2}, \quad V_{C}(q)=-\frac{g^{2}}{2 \cosh ^{2} q}+\frac{g^{2}}{2} .
$$

The quantum eigenvalues and eigenfunctions are:

$$
E_{n}=g n \hbar-n^{2} \hbar^{2} / 2, \quad \mathcal{E}_{n}=g n, \phi_{n}(q)=(\cosh q)^{n} P_{n}^{(\alpha, \alpha)}(\tanh q), \alpha \equiv g / \hbar-n>0,
$$

in which $P_{n}^{(\alpha, \beta)}(x)$ is the Jacobi polynomial of degree $n$. The classical equilibrium point is the origin $\bar{q}=0$ and the classical eigenfunction and the Hessian $\widetilde{W}(12)$ are:

$$
\varphi_{n}(q)=n ! \lim _{\hbar \rightarrow 0} \hbar^{n} \phi_{n}(q)=g^{n}(\sinh q)^{n}, \quad-\widetilde{W}=g .
$$

It is easy to verify (17) and the Propositions 1-3. For integer $g / \hbar, V(q)$ (26) (without the constant term) is the reflectionless potential corresponding to a $\mathrm{KdV}$ soliton. In both examples, $\varphi_{1}$ is the elementary excitation.

Throughout this Letter we have assumed that the prepotential $W$ is independent of the Planck's constant $\hbar$, for simplicity of the presentation. The main content of this Letter is valid even if $W$ depends on $\hbar$, so long as $\lim _{\hbar \rightarrow 0} W=W_{0}$ is well-defined. A celebrated example that $\lim _{\hbar \rightarrow 0} W$ diverges is the hydrogen atom, for which the classical equilibrium does not exist. In this case the quantum-classical correspondence does not make sense and the present formulation does not apply.

\section{Acknowledgements}

We thank David Fairlie and Cosmas Zachos for useful discussion. I.L. is a post-doctoral fellow with the F.W.O.-Vlaanderen (Belgium).

\section{References}

[1] E. Corrigan and R. Sasaki, "Quantum vs classical integrability in Calogero-Moser systems", J. Phys. A35 (2002) 7017-7062, hep-th/0204039; S. Odake and R. Sasaki, "Polynomials associated with equilibrium positions in Calogero-Moser systems," J. Phys. A35 (2002) 8283-8314, hep-th/0206172; O. Ragnisco and R. Sasaki, "Quantum vs classical integrability in Ruijsenaars-Schneider systems", Preprint YITP-03-09, hep-th/0305120 
[2] F. Calogero, "Solution of the one-dimensional $N$-body problem with quadratic and/or inversely quadratic pair potentials", J. Math. Phys. 12 (1971) 419-436; B. Sutherland, "Exact results for a quantum many-body problem in one-dimension. II", Phys. Rev. A5 (1972) 1372-1376; J. Moser, "Three integrable Hamiltonian systems connected with isospectral deformations", Adv. Math. 16 (1975) 197-220.

[3] I. Loris and R. Sasaki, "Quantum \& Classical Eigenfunctions in Calogero and Sutherland systems", Kyoto preprint YITP-03-51, hep-th/0308052, to be published in J. Phys. A.

[4] A. J. Bordner, N. S. Manton and R. Sasaki, "Calogero-Moser models V: Supersymmetry and Quantum Lax Pair", Prog. Theor. Phys. 103 (2000) 463-487, hep-th/9910033 S. P. Khastgir, A. J. Pocklington and R. Sasaki, "Quantum Calogero-Moser Models: Integrability for all Root Systems", J. Phys. A33 (2000) 9033-9064, hep-th/0005277.

[5] G. J. Heckman and E. M. Opdam, "Root systems and hypergeometric functions I-IV", Comp. Math. 64 (1987) 329-352; 353-373; 67 (1988) 21-49; 191-209.

[6] See, for example, F. Cooper, A. Khare and U. Sukhatme, "Supersymmetry and quantum mechanics," Phys. Rept. 251 (1995) 267-385.

[7] G. Darboux, "Sur une proposition relative aux équation linéaires, Compte Rendu Acad. Sci. 94, (1882) 1456-1459.

[8] G. Pöschl and E. Teller, "Bemerkungen zur Quantenmechanik des anharmonischen Oszillators", Z. Physik 83 (1933) 143-151; see for a different treatment, T. Curtright, D. Fairlie and C. K. Zachos, "Features of time-independent Wigner functions," Phys. Rev. D58 (1998) 025002, hep-th/9711183.

[9] A. M. Perelomov, "On frequencies of small oscillations of some dynamical systems associated with root systems", J. Nonlinear Math. Phys. 10 (2003) 103-109, math-ph/0205039 